\title{
Laser-based precise measurement of tailor welded blanks: a case study
}

\author{
Yuanyuan Zou ${ }^{1,2}$, Kezhu Zuo ${ }^{3}$, Honghai Liu ${ }^{4}$, and Dalin Zhou ${ }^{4}$ \\ 1.School of Mechanical Engineering, Shenyang Jianzhu University 2.National-Local \\ Joint Engineering Laboratory of NC Machining Equipment and Technology of \\ High-Grade Stone 3.Vision and Intelligent System, Micro-Nano Automation Institute \\ 4.School of Computing, University of Portsmouth
}

\begin{abstract}
Precise measurement of tailor-welded blanks is crucial for quality control of laser welding. The difficulty in measuring the seam profile of similar gage tailorwelded blanks lies in lacking of solutions to locate their feature points such as those representing the laser stripe. A laser sensorbased method is proposed to measure tailor-welded blanks based on its laser stripe and texture features, from which the proposed algorithm is employed to extract the feature points for seam profile assessment. The algorithm is evaluated on a laser-based weld seam measurement system, the experiment results show that the proposed method measures the tailor-welded blanks with high accuracy and is suitable for online inspection.
\end{abstract}

\section{Introduction}

A tailor-welded blank (TWB) is composed of two or more dissimilar sheet metals with different shapes, strengths, materials, or thicknesses that are butt-welded together before being formed [1]. Recently, a tailored blank laser welding has been widely used in the automotive, aerospace, electronics, and heavy manufacturing industries due to its high speed and high precision [2-4]. TWBs are replacing conventional sheet metal blanks in a rapidly increasing number of these applications [5]. In addition, TWBs are not limited to using dissimilar sheet metals that are butt-welded together, the similar gage sheet metals are used for TWBs to increase material utilization and reduce the number of forming and material handling operations [6]. Measurement of the weld seam profile is critical for ensuring high welding quality of the laser welding [7]. Online measurement can provide weld quality information that can be used to optimize welding parameters and reduce cycle time and material waste by terminating the welding process when weld defects are detected [8]. Accordingly, many methods have been presented for inspecting the quality of weld seams in laser welding, including electromagnetic acoustic transducers [9], laser vision sensors [10-12], and magnetic flux leakage detection systems [13]. Among these methods, the laser vision sensor is noncontact and can be easily mounted on the rear of a welding torch to perform online weld seam inspection after completion of the welding process. Some laser vision sensors have been developed for weld seam inspection, and most of them 
have been used to measure the weld seam profile according to only the feature points of the laser stripe [14-16]. However, in measuring the seam profile for similar gage-tailored blanks, it is difficult to identify the feature points because the weld seam is small and the laser stripe feature points are not obvious. To solve this problem, a method for measuring the weld seam profile based on a laser stripe and texture features is proposed in this paper. The rest of this paper is organized in the following manner. A laser sensorbased method is proposed to measure tailor-welded blanks based on its laser stripe and texture features, from which the proposed algorithm is employed to extract the feature points for seam profile assessment in Section 2. In Section 3, the structure and configuration of the laser vision sensor used to capture a grayscale image of the weld seam with a laser stripe are described and analyzed, and a direct calibration method for calibrating the laser vision sensor is discussed. In Section 4, a weld seam measurement system is built, and experiments are carried out. The experimental results for the weld seam measurements are presented. This paper is concluded in Section 5.

\section{The Proposed Method}

The weld seam image of the similar gage tailorwelded blanks contains the weld seam region, the sheet metals, and the heat-affected zone. Figure 1 is an image of the weld seam which the thickness of tailored blanks is $1.0 \mathrm{~mm}$. In the measurement of the seam profile for similar thickness tailored blanks, it is difficult to identify the feature points only according to the laser stripe because the weld seam is small and the turning points of the laser stripe are not obvious. The texture features in the weld seam region and the non-weld seam region have obvious differences. Thus, a laser sensorbased method is proposed based on its laser stripe and texture features for extracting the feature points. In this section, the image processing algorithm is explained. The flow chart of the algorithm is shown in Fig. 2. The algorithm includes the weld seam image segmentation based on texture features, the feature point extraction, and the dimensional parameters calculation.

\subsection{Weld seam image segmentation based on texture features}

Texture features of weld seam image First, a ROI of the weld seam image in a given area is selected to reduce the computational load of the measurement process. And then, the ROI image needs to be divided into n sub-images with a size of $\mathrm{w} x \mathrm{~h} 0$ to segment the weld seam region effectively and reduce the processing time. The equation for $\mathrm{n}$ is as follows:

$n=\frac{\text { col } \times \text { row }}{\mathrm{w} \times \mathrm{h}}$

where col is the width of the image and row is the height of the image, $\mathrm{w}$ is the width of the sub-image, and $h$ is the height of the sub-image. The subimage needs to be large enough that the texture features of the sub-image can 


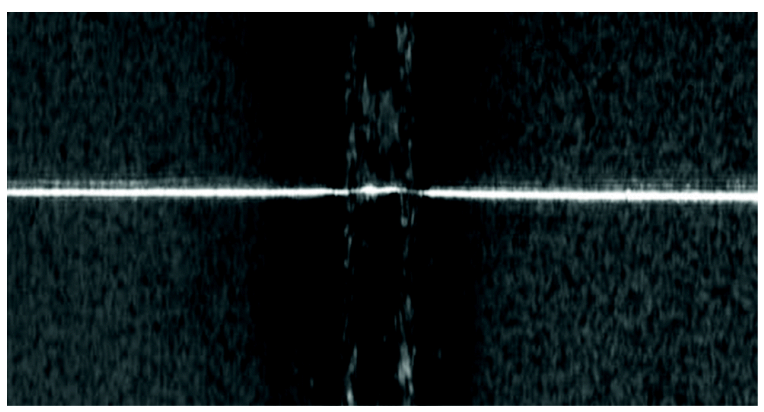

Fig. 1. An image of the weld seam

adequately reflect the texture features of the weld seam. Meanwhile, if the subimage is too large, the recognition error will increase. Therefore, an appropriate size for the sub-image should be chosen. In this paper, the size is determined by the experiments, and the appropriate size is 20 pixels x 40 pixels. Consequently, the texture features of the weld seam image need to be extracted. There are various methods for analyzing the texture features of an image, such as the structural texture analysis method, the spectral method, and the texture model method [1719]. These methods have characteristics that are not intended for analyzing the texture feature of weld seam images. In view of the natural texture of a weld seam for analysis, the method used in this paper is the statistical method that contains grayscale histogram and gray-level co-occurrence matrix (GLCM). The texture features of different regions of weld seam images are calculated based on grayscale histogram, such as the mean, standard deviation, smoothness, third moment, consistency, and entropy, which can provide statistics on the texture properties of roughness and smoothness. Three sub-images with a size of 20 pixels $\mathrm{x} 40$ pixels are selected from the weld seam region, the sheet metals region, and the heat-affected zone for calculating the six statistical features mentioned above. The results are shown in Table 1 . The results show that the texture features calculated based on grayscale histogram are different for the weld seam region and the non-weld seam region.

\begin{tabular}{|c|c|c|c|c|c|c|}
\hline Region & Mean & Standard deviation & Smoothness & Third moment & Consistency & Entropy \\
\hline Weld seam region & 90.18 & 5.56 & $4.74 \mathrm{e}-04$ & $6.64 \mathrm{e}-04$ & 0.06 & 4.34 \\
\hline Metal sheet & 33.92 & 2.16 & $7.20 \mathrm{e}-05$ & $6.47 \mathrm{e}-05$ & 0.16 & 2.97 \\
\hline Heat-affected zone & 61.33 & 20.42 & $6.30 \mathrm{e}-03$ & 0.10 & 0.02 & 5.90 \\
\hline
\end{tabular}

The GLCM is a matrix where the number of rows and columns is equal to the number of grayscale level L in an image [20]. The matrix element is also represented as $\mathrm{p}\left(z_{i}, z_{j}\right)$, which contains the second-order probability values for changes between grayscale level $z_{i}$ and $z_{j}$ at a distance $\mathrm{d}$ and a particular angle 


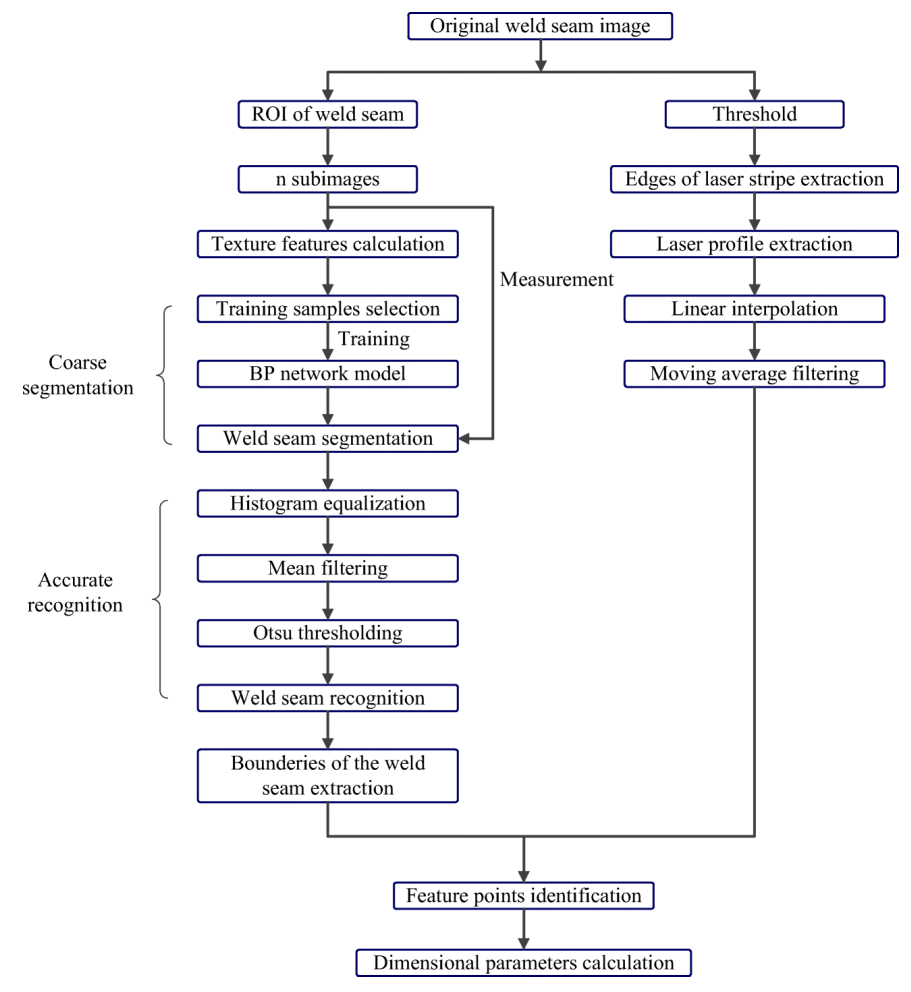

Fig. 2. Flow chart of the algorithm

$\theta$. The mean and standard deviations for the rows and columns of the matrix are shown as follows:

$$
\begin{aligned}
m_{r} & =\sum_{i=0}^{L-1} z_{i} \sum_{j=0}^{L-1} p\left(z_{i}, z_{j}\right) \\
m_{c} & =\sum_{i=0}^{L-1} z_{j} \sum_{j=0}^{L-1} p\left(z_{i}, z_{j}\right) \\
\sigma_{r}^{2} & =\sum_{i=0}^{L-1}\left(z_{i}-m_{r}\right)^{2} \sum_{j=0}^{L-1} p\left(z_{i}, z_{j}\right) \\
\sigma_{c}^{2} & =\sum_{i=0}^{L-1}\left(z_{j}-m_{c}\right)^{2} \sum_{j=0}^{L-1} p\left(z_{i}, z_{j}\right)
\end{aligned}
$$

Various texture features are extracted from the GLCM. Among these texture features, six texture features, which are defined as the following equations, are analyzed in this paper.

$$
\begin{aligned}
& \text { Energy: } V_{1}=\sum_{i=0}^{L-1} \sum_{j=0}^{L-1}\left[p\left(z_{i}, z_{j}\right)\right]^{2} \\
& \text { Contrast: } V_{2}=\sum_{i=0}^{L-1} \sum_{j=0}^{L-1}\left(z_{i}-z_{j}\right)^{2} p\left(z_{i}, z_{j}\right)
\end{aligned}
$$


Homogeneity: $V_{3}=\sum_{i=0}^{L-1} \sum_{j=0}^{L-1} \frac{p\left(z_{i}, z_{j}\right)}{1+\left|z_{i}-z_{j}\right|}$

Entropy: $V_{4}=-\sum_{i=0}^{L-1} \sum_{j=0}^{L-1} p\left(z_{i}, z_{j}\right) \times \log _{2} p\left(z_{i}, z_{j}\right)$

Correlation: $V_{5}=\sum_{i=0}^{L-1} \sum_{j=0}^{L-1} \frac{\left(z_{i}-m_{r}\right)\left(z_{j}-m_{c}\right) p\left(z_{i}, z_{j}\right)}{\sigma_{r} \sigma_{c}}$

Maximum probability: $V_{6}=\max \left(p\left(z_{i}, z_{j}\right)\right)$

Because the weld seam is in the vertical direction of the image, the angle is chosen as $\theta=90$. The six texture features are calculated at different distances $\mathrm{d}$, and the results are shown in Fig. 3, where the distance $d$ is the abscissa and the texture feature is the ordinates. When the value of $d$ is equal to 10 , most of the texture features of the weld seam region and the non-weld seam region are obviously different.
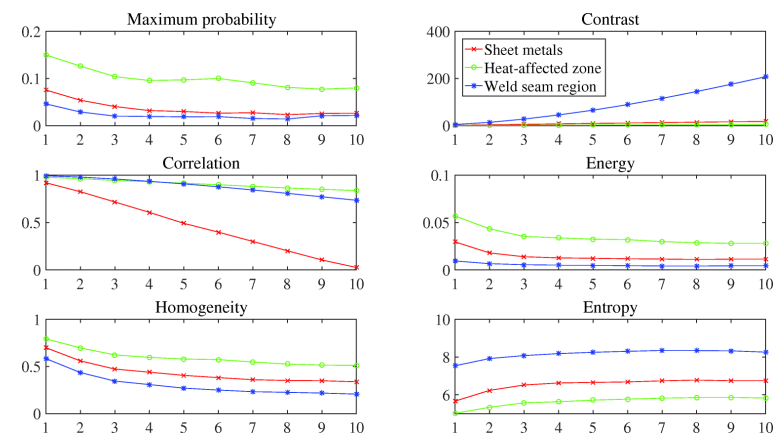

Fig. 3. Texture features of GLCM at different distances d

Weld Seam Segmentation It is important to accurately segment the weld seam region to extract the feature points of the weld seam. In this paper, a two-step segmentation method is proposed. The backpropagation (BP) neural network model is trained to roughly identify the region of the weld seam. Then, an accurate method is used for weld seam recognition.

The BP neural network consists of artificial neurons distributed in different layers. The basic structure of the BP network comprises an input layer, an output layer, and some hidden layers. Before training the network, the appropriate parameters of the network should be designed to obtain better recognition results.

The 12 texture features are the inputs of the network, so the number of neurons in the input layer is 12 . The number of neurons in the output layer is 2 . The more hidden layers that exist in a network, the more time it takes to train the network. The number of hidden layers is 1 because the BP network with three layers can effectively achieve an unknown nonlinear function. The number 
of neurons in the hidden layer is 7 . The sigmoid function is chosen as the action function, and the learning rate is 0.01 .

The training samples used to train the BP network are obtained as follows. First, four images are randomly selected from the image series. Sub-images of the non-weld image region and sub-images of the weld seam region are selected from the ROI of every image. The non-weld image region contains the heat-affect zone and the sheet metals. The size of the sub-image is 20 pixels $\mathrm{x} 40$ pixels. When the area of the weld seam region is more than $2 / 3$ of the area of the sub-image, the sub-image is defined as the weld seam region. Second, the texture features are calculated for every sub-image, and a matrix A1 is obtained. The training samples are obtained using the normalization process to A1. Finally, the nonweld seam region is represented as 0 , and the weld seam region is represented as 1. The output vector of the network is a matrix represented as A2. The BP network is trained, and the training time is $1 \mathrm{~s}$.

The trained BP neural network model is used to identify the region of the weld seam. The recognition steps are as follows.

Step 1:: The image is divided into sub-images of 20 pixels x 40 pixels, and the texture features of every sub-image are calculated to form matrix B1.

Step 2:: Normalization processing is used on matrix B1, and then, the obtained matrix is used as the input of the trained BP network. The output matrix is matrix $\mathrm{B} 2$.

Step 3:: The elements of matrix B2 are rounded, which element 0 belongs to the non-weld seam region, and element 1 belongs to the weld seam region. After the segmentation, there are usually some sub-images being segmented incorrectly. Therefore, processing to reduce false classification errors is needed. A threshold is used for image thresholding. After threshold segmentation, the largest connected region is obtained, which is the weld seam region. The subimage surrounded by sub-images of the weld seam region but identified as a non-weld seam region are filled, and the sub-images in the edge of the largest connected region are processed, such as filling the depressions and removing the protruding sub-images. Then, the weld seam region is identified.

An accurate recognition algorithm is proposed to identify the weld seam region and shown below. The weld seam region identified above is as the ROI.

Then, the histogram equalization is carried out for the ROI. Consequently, the image is smoothed with a mean filter operator of $301 \times 1$ due to the uneven distribution of grayscale. For the smoothed image, the Otsu thresholding is applied. Finally, the grayscale projection in the vertical direction of the image is calculated, and the left and right boundaries of the weld seam are extracted based on the extreme points.

\subsection{Feature Point Extraction}

The dimensional parameters of the weld seam are measured using the left and right feature points of the weld seam. The feature points are defined as the intersection points of the laser stripe profile and the left and right weld seam boundaries. 
The algorithm for extraction of the laser stripe profile is presented first. Laser tailored blank welding is a welding technology with high speed, so a higher image processing speed is needed to satisfy the requirement. Some algorithms have been developed for profile extraction based on intensity distribution using methods such as intensity distribution fitting, center of gravity, and multi-peak detection $[21,22]$. Among these methods, the center of gravity method is commonly used due to its fast speed and high precision. However, the reflections of the laser stripe are different from the smooth metal surface and the weld seam surface, which causes confusion; for example, the width of the laser stripe is different in that the laser stripe is usually ruptured and there are burrs in the extracted laser profile. An improved center of gravity method is applied in this paper according to the width of the laser stripe to achieve the robustness of stripe profile extraction with high precision. First, the stripe figure in the image is detected by thresholding, and the up and down edges of the laser stripe are extracted by scanning the segmented laser stripe. Then, the location and light intensity of each pixel on the extracted edges can be used to identify the row coordinates of the center points of the laser stripe. The equation is as follows:

$$
y_{j}=\frac{\sum_{i=w_{j}}^{k_{j}} f(i, j) \cdot i}{\sum_{i=w_{j}}^{k_{j}} f(i, j)}
$$

where $f(i, j)$ represents the light intensity of the pixel in column $j$ and row $i$ after the image is median filtered, $w_{j}$ and $k_{j}$ represents the row coordinate of the pixel on the up and down edge of laser stripe in column $\mathrm{j}$, respectively, and $y_{j}$ represents the row coordinate of the center point in column $\mathrm{j}$. Then, the profile of the laser stripe is extracted, and the coordinates of the profile are obtained.

Then, for the extracted profile that is found ruptured or has burrs, the processing algorithm is performed in the following manner. First, the search is carried out along the extracted laser profile. When the column coordinates difference of the adjacent pixel on the laser profile is more than 1 pixel, the linear interpolation of the nearest neighbor points is used to mend the ruptured profile to obtain a continuous profile. Then, a one-dimensional moving average filter is applied for removing disturbing burrs. The width of the filter window is selected as 12 in the experiments.

Finally, the intersection points of the extracted laser profile and the left and right boundaries of the weld seam are identified as the feature points. The dimensional parameters of the weld seam can be calculated by the feature points.

\subsection{Dimensional Parameters Measurement}

The feature points of the laser profile are extracted as p1 and p2 which can be used to calculate the dimensional parameters of weld seam, such as the bead width, mismatch, concavity, or convexity, as shown in Fig. 4. The definition of the dimensional parameters is as follows. The bead width is defined as the horizontal distance between $\mathrm{p} 1$ and $\mathrm{p} 2$. The mismatch is defined as the difference in height of the two parent metal surfaces being welded. Weld concavity is defined as the 
underfill (or suck back) of metal in the weld causing the weld surface to be below flush with the parent metal surfaces. Weld convexity is defined as the solidified, overfilled weld metal causing the weld surface to be above flush with the parent metal surfaces.

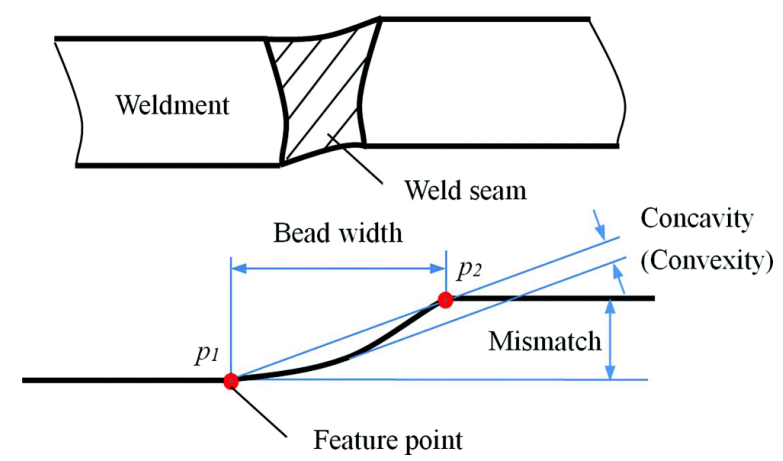

Fig. 4. Definition of dimensional parameters of the weld seam

During welding, weld images sometimes are corrupted with weld spatter, so the measurement results are affected by these disturbances. The false results are rejected using temporal and spatial continuity constraints. Subsequently, a supervising mechanism is adopted to give a recommended measurement result if the false result is rejected. A given array will be used to save five history measurement results, and the mean of the history measurement results is calculated according to the array. When the difference between the mean and the current result is more than the set threshold of $0.2 \mathrm{~mm}$, the mean will substitute for the current measurement result.

\section{Laser Vision Sensor}

\subsection{Laser Vision Sensor Description}

The laser vision sensor is used to capture the weld seam images. It consists of a laser projector, a camera module, and a LED ring light, as shown in Fig. 5a. The laser projector is used to generate a laser stripe that projects onto the weld seam. The camera module includes a focus lens and a Camera Link camera with a complementary metaloxidesemiconductor (CMOS) image sensor. The CMOS camera is used to record a 1024 pixels x 1024 pixels grayscale image at the same point on the weld seam where the laser stripe is located. Thus, the image captured by the camera is a grayscale image with a laser stripe. The principle of the measurement is primarily based on the laser triangulation technique.

The laser vision sensor is mounted on the rear of the welding torch and above the weldment. During welding, the sensor scans the weld seam and captures the 

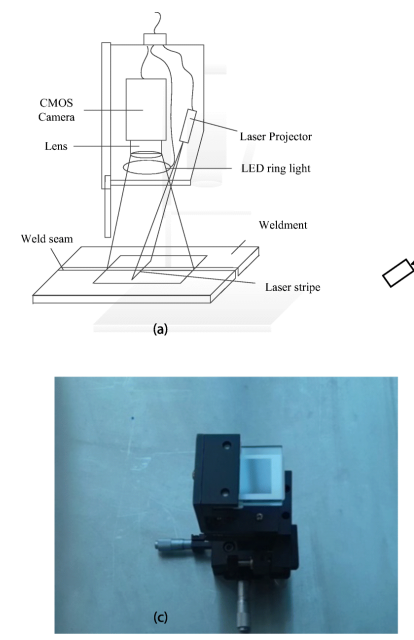

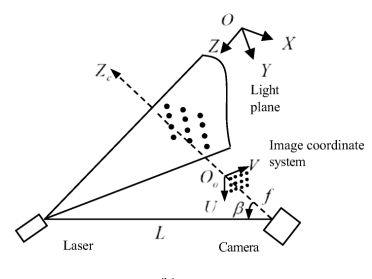

(b)

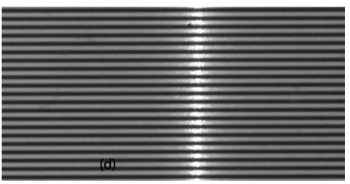

Fig. 5. a Diagram of the laser vision sensor; b the principle of the direct calibration method; c photo of the calibration target; $d$ stripes on a calibration plate

grayscale images of the weld seam with a laser stripe. The acquired images are transferred to the image processing module for extracting the feature points of the weld seam and calculating the profile dimensional parameters.

\subsection{Principle of Calibration}

To calibrate the laser vision sensor of small field of view applied in weld seam measurement with high accuracy and high efficiency, a direct calibration method based on a support vector machine (SVM) is discussed. The principle of the direct calibration method is shown in Fig. 5b.

OoUV is the image coordinate system. OXYZ is the world coordinate system. The image coordinate $(\mathrm{u}, \mathrm{v})$ of an arbitrary point in the light plane has a relationship to its world coordinates $(\mathrm{x}, \mathrm{y}, \mathrm{z})$. The image plane is in the OYZ plane of the world coordinate system. Therefore, the system only needs to build the relationship between the image coordinates $(\mathrm{u}, \mathrm{v})$ and the world coordinates $(\mathrm{y}, \mathrm{z})$. The world coordinates of a measurement point can be obtained when the measurement is performed according to the relationship of the image coordinates and the world coordinates.

\subsection{Calibration Method}

A direct calibration method is proposed for defining the differential measurements between the image plane and the weld frame based on direct mapping models. The models are built according to the image coordinates and the threedimensional coordinates of some feature points based on an SVM. Two models 
for the coordinates y and $\mathrm{z}$ in space are established to calibrate the laser vision sensor.

First, a calibration target is designed to calibrate the laser vision sensor. A calibration plate is adopted with high-precision equi-spaced black and white stripes. The width of the stripe is determined by the measurement accuracy of the laser vision sensor as well as the field of view of the sensor. The calibration plate is mounted on a three-dimensional precision translation stage. The position and attitude of the calibration plate are adjusted to ensure that the measured surface includes the stripes perpendicular to the vertical direction of the precision translation stage and that the direction of the stripes are parallel to the horizontal movement direction of the precision translation stage. The photo of the calibration target is shown in Fig. 5c. And the image of the stripes on a translation stage is shown in Fig. 5d.

The calibration is carried out by using feature points on the extracted laser stripe. The feature points are defined as the intersection points of the laser stripe centerline and the white stripe centerlines. When the laser projector projects the laser stripe perpendicular to the black and white stripes on the calibration plate, the reflected images of the laser strip are captured by the CMOS camera as along with the black and white stripes. The reflected images are processed, and the feature points are extracted.

First, the region of interest (ROI) that contains the laser stripe and the ROI that contains the black and white stripes of the calibration plate are set and extracted respectively. The algorithm can automatically determine the ROIs. Second, the centerline of the laser stripe and the centerlines of the white stripes on the calibration plate are extracted based on the intensity distribution. In each laser stripe profile, a Gaussian profile of the intensity of the pixels versus their location is generated, and a Gaussian filter is used to smooth the profile. Then, the peak of the Gaussian profile is chosen as the pixel location for the laser stripe centerline extraction. In each row of the ROI that contains black and white stripes, the sum of the intensity value is calculated, and the max values are chosen as the pixel locations primarily for white stripe centerline extraction. To accurately extract the white stripe centerline, a sliding window of 33 is used to search along the extracted white stripe centerline through the grayscale centroid method. Consequently, the centerline of the laser stripe and the centerlines of the white stripes are fitted by least square fitting. Finally, the intersection points of the laser stripe centerline and the white stripe centerlines are recognized, and the subpixel image coordinates of the feature points are obtained.

The feature points in different positions of the light plane need to be recorded for building the direct mapping models. The calibration method is as follows:

1) The three-dimensional precision translation stage is adjusted to ensure that the laser stripe is on the initial measurement position.

2) A world coordinate system that originates at the calibration target is built. The origin position is defined as the intersection point of the laser stripe centerline and the white stripe centerline on the end of the calibration plate. Axis $\mathrm{Z}$ is defined as the vertical movement direction of the three-dimensional precision 
translation stage. Axis $\mathrm{X}$ is defined as the length direction of the black and white stripes on the calibration plate. Axis $\mathrm{Y}$ is defined as the direction perpendicular to the length direction of the black and white stripes on the calibration plate. The world coordinates of the feature points are calculated by the origin position, the width of the stripes, and the indication value in different axis directions of the three-dimensional precision translation stage.

3) The images are captured for calibration. A calibration image is captured at the initial position. Then, the three-dimensional precision translation stage is adjusted in a fixed step along the Z-direction. One calibration image will be captured when every adjustment is carried out until the image collection process is finished.

4) The calibration images are processed for extracting the feature points by the algorithm proposed above. The subpixel image coordinates $(\mathrm{u}, \mathrm{v})$ of the feature points are obtained.

5) The direct mapping models are established between image coordinates and three-dimensional coordinates based on SVM. The image coordinates $(\mathrm{u}, \mathrm{v})$ of the entire extracted feature points are selected as the network input, the world coordinate $\mathrm{y}$ is selected as the output, and the SVM model of the coordinate $\mathrm{y}$ is established. Then, the image coordinates $(\mathrm{u}, \mathrm{v})$ of the entire extracted feature points are selected as the network input, the world coordinate $\mathrm{z}$ is selected as the output, and the SVM model of the coordinate $\mathrm{z}$ is also established.

In the measurement, when the image coordinates of an arbitrary point are input to the model of coordinate $y$ and the model of coordinate $\mathrm{z}$, three-dimensional coordinates of the point can be obtained from the models, and the vision sensor can be calibrated.

\section{Experiments And Analysis}

Experiments are conducted to verify the algorithms feasibility and precision. First, a calibration precision validation experiment is conducted to verify the calibration precision. The width of the stripes in the calibration plate is 0.05 $\mathrm{mm}$ and the accuracy is $0.01 \mathrm{~mm}$. The resolution in three directions of the threedimensional precision translation stage is $0.002 \mathrm{~mm}$. One hundred test points are selected randomly in the light plane. The image coordinates of the test points are obtained by image processing. And they are fed into the SVM models. The world coordinate $\mathrm{y}$ and $\mathrm{z}$ of these test points are predicted from the models. By comparing the predicted world coordinates with the real-world coordinates which are calculated according to the indication value in different axis directions of the three-dimensional precision translation stage, the accuracy of the calibration method is calculated. Table 2 presents the results, in which the mean absolute error is $0.0196 \mathrm{~mm}$ in $\mathrm{Y}$-direction and $0.0138 \mathrm{~mm}$ in Z-direction. And then the dimensional parameters of the weld seam, including weld seam width, mismatch, concavity, or convexity are measured. The experimental equipment for weld seam measurement is shown in Fig. 6, which consists of a laser vision sensor, an image processing and control system, and laser welding equipment. In 
the laser welding equipment, a positioning and clamping mechanism is adopted to make the position of the weld blanks fixed during the welding process. The laser vision sensor is mounted on the rear of the welding torch for capturing the weld seam images. And the laser stripe of the laser vision sensor is adjusted perpendicularly to the weld seam to make the weld seam region upright in sensing images. The laser vision sensor is mounted on the rear of the welding torch for capturing the weld seam images and controlled by the image processing and control system. Then, the images are processed, and the dimensional parameters of the weld seam are obtained. Three sets of workpieces are selected for tailored blank laser welding and for the measurement experiments. The blank thickness is shown in Table 3.

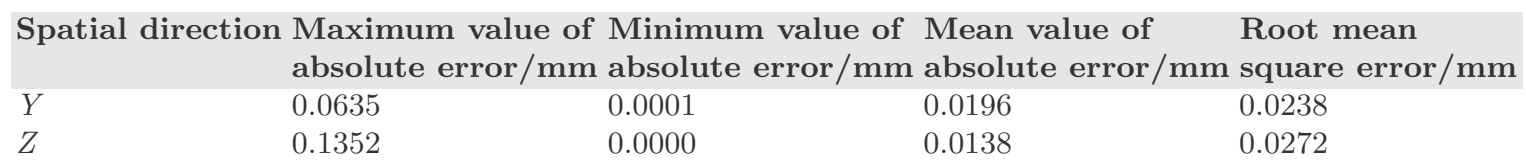

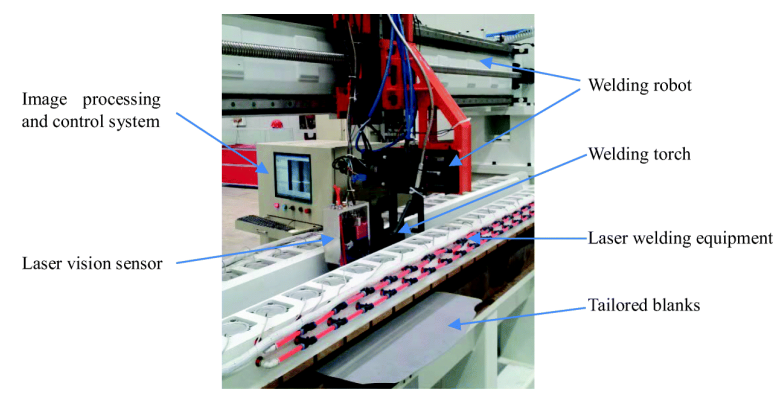

Fig. 6. Experimental equipment for weld seam measurements

$\begin{array}{ll}\text { Serial number Tailor-welded blanks thickness } / \mathbf{m m} \\ 1 & 1.01 .0 \\ 2 & 0.70 .7 \\ 3 & 1.21 .2\end{array}$

In this paper, the BP neural network is used as a primarily segmentation method for weld seam recognition. To evaluate the efficiency of the BP neural network for segmenting the weld seam region, the K-means algorithm is used to compare with it. The 12 texture features are used as input to K-means algorithm, and output is the segmenting result. As shown in Fig. 7, the performances of 
the BP neural network are better than the K-means algorithm for all three sets weld seam.
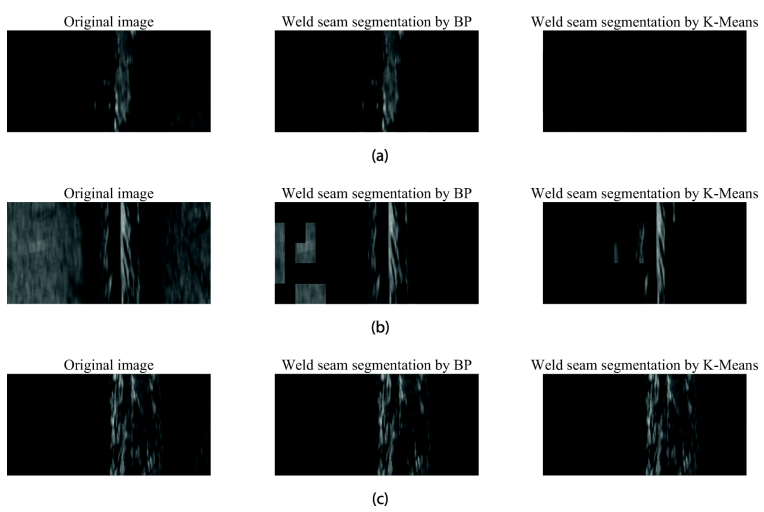

Fig. 7. The weld seam primarily segmentation comparison by BP neural network and K-means. a The first set; $b$ the second set; $c$ the third set

As an example, the weld seam coarse recognition result for Fig. 1 is shown in Fig. 8.

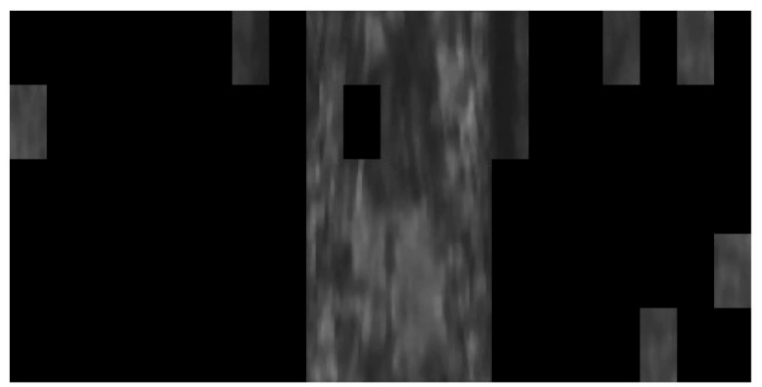

Fig. 8. Recognition result from the BP neural network

The result shows that the weld seam region can be primarily identified. And then the proposed accurate recognition algorithm is used to identify the weld seam region and the results are shown in Fig. 8. The weld seam region identified above is as the ROI shown in Fig. 9a. Then, the histogram equalization is carried out for the ROI, and the result is shown in Fig. 9b, which clearly shows the weld seam display. Consequently, the image is smoothed and the result is shown in Fig. 9c. For the smoothed image, the Otsu thresholding is applied. Finally, the grayscale projection in the vertical direction of the image is calculated, and the left and right boundaries of the weld seam are extracted based on the extreme 
points. The result is shown in Fig. 9d. Figure 10 shows the recognition results of the feature points of the three sets weld seam.

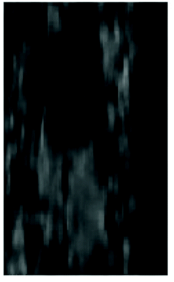

(a)

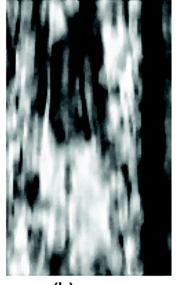

(b)

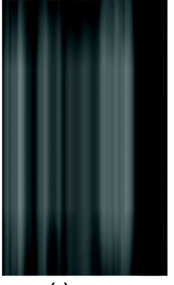

(c)

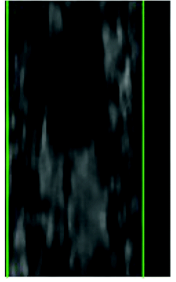

(d)

Fig. 9. Accurate recognition of the weld seam boundaries. a ROI; b histogram equalization; c mean smoothing; d weld seam boundary extraction
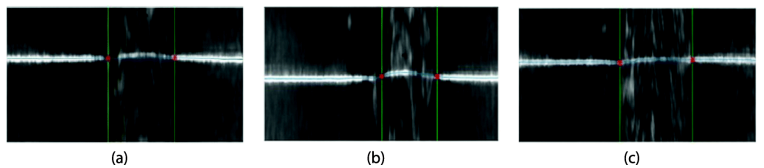

Fig. 10. Feature point recognition results of the weld seam. a The first set; $b$ the second set; $c$ the third set

In Fig. 11, the weld seam width measurement value and the true value are compared respectively for the three sets weld seam, which the measurement value is obtained from a sequence of frames in tailored blank laser welding of similar gage blanks. To verify the precision of the measurement, manual measurement is used as a precise measurement method by measuring the weld bead at the same positon as the laser stripe projected. A vernier caliper is used by sampling the weld bead ten times at each position. The accuracy of the vernier caliper is $0.01 \mathrm{~mm}$. The value measured by this method is defined as the true value, which can be used to compare with the results measurement.

In this paper, three indicators are adopted for error analysis: root mean square error (RMSE), mean value of the absolute error, and mean value of the relative error. In Table 4, the RMSE of the first set is $0.12 \mathrm{~mm}$, and the mean value of the relative error is $9.99 \%$. The RMSE of the second set is $0.064 \mathrm{~mm}$, and the mean value of the relative error is $6.36 \%$. The RMSE of the third set is $0.054 \mathrm{~mm}$, and the mean value of the relative error is $3.81 \%$.

Figure 11b shows that the measured weld seam width of the second set is less than the true value due to the interference of the heat-affected zone, as shown in Fig. 10b. Figure 11c shows that the weld seam width measurement value of the third set is very close to the true value. The texture feature of the weld seam region is obvious compared to that of the non-weld region in Fig. 10c. Mismatch, 


\begin{tabular}{|c|c|c|c|c|}
\hline Serial number & $\begin{array}{l}\text { Root mean square } \\
\text { error }(\mathrm{mm})\end{array}$ & $\begin{array}{l}\text { Mean value of } \\
\text { absolute error }(\mathrm{mm})\end{array}$ & $\begin{array}{l}\text { Mean value } \\
\text { of relative error }(\%)\end{array}$ & $\begin{array}{l}\text { Image processing } \\
\text { time per frame ( } \mathrm{ms})\end{array}$ \\
\hline 1 & 0.120 & 0.109 & 9.99 & 789 \\
\hline 2 & 0.064 & 0.056 & 6.36 & 831 \\
\hline 3 & 0.054 & 0.043 & 3.81 & 796 \\
\hline
\end{tabular}

concavity, or convexity can also be measured. The processing time per frame can satisfy the requirement of the quality inspection of the weld seam.

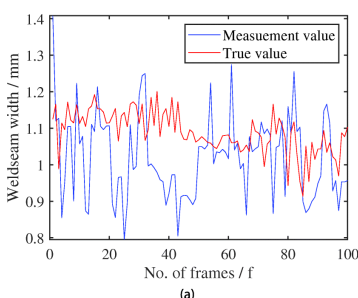

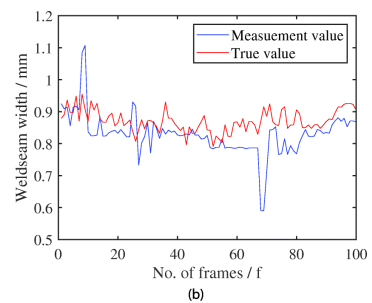

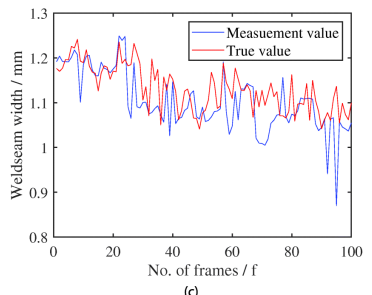

Fig. 11. The weld seam width measurement comparison. a The first set; $b$ the second set; $c$ the third set

\section{Conclusions}

In this research, a laser sensorbased method for measuring the weld seam profile of similar gage-tailored blanks based on laser stripe and texture features is put forward, from which the proposed algorithm is employed to extract the feature points for seam profile assessment. The conclusions are addressed as follows:

1. The new image processing algorithm for segmenting the weld seam region and extracting the feature points of the weld seam are proposed, and the profile dimensional parameters including weld seam width, mismatch, concavity, or convexity can be obtained.

2. A detailed structure and configuration of a laser vision sensor are presented that can capture a grayscale image of a weld seam with a laser stripe. And a direct calibration method is discussed for calibrating the laser vision sensor with high accuracy. 
3. Experimental results show that the dimensional parameters of the weld seam can be measured with satisfactory results in tailored blank laser welding, and it is suitable for online inspection.

\section{References}

1. Assunco E, Quintino L, Miranda R (2010) Comparative study of laser welding in tailor blanks for the automotive industry. Int J Adv Manuf Technol 49(1-4):123131

2. Merklein M, Johannes M, Lechner M, Kuppert A (2014) A review on tailored blanks-production, applications and evaluation. J Mater Process Tech $214(2): 151164$

3. Sinke J, Iacono C, Zadpoor AA (2010) Tailor made blanks for the aerospace industry. Int J Mater Form 3(1 Supplement):849852

4. Anand D, Chen DL, Bhole SD, Andreychuk P, Boudreau G (2006) Fatigue behavior of tailor (laser)-welded blanks for automotive applications. Mat Sci Eng A 420(12):199207

5. Nguyen HC, Lee BR (2014) Laser-vision-based quality inspection system for smallbead laser welding. Int J Precis Eng Man 15(3):415423

6. Abdullah K, Wild PM, Jeswiet JJ, Ghasempoor A (2001) Tensile testing for weld deformation properties in similar gage tailor welded blanks using the rule of mixtures. J Mater Process Tech 112(1):9197

7. You DY, Gao XD, Katayama S (2014) Multisensor fusion system for monitoring high-power disk laser welding using support vector machine. IEEE T Ind Inform 10(2):12851295

8. Li Y, Li YF, Wang QL, Xu D, Tan M (2010) Measurement and defect detection of the weld bead based on online vision inspection. IEEE T Instrum Meas 59(7):18411849

9. Salzburger HJ (2010) EMATs and its potential for modern NDE-state of the art and latest applications-. Ultrasonics Symposium

10. Stavridis J, Papacharalampopoulos A, Stavropoulos P (2018) Quality assessment in laser welding: a critical review. Int J Adv Manuf Technol 94(1):123

11. Schreiber D, Cambrini L, Biber J, Sardy B (2009) Online visual quality inspection for weld seams. Int J Adv Manuf Technol 42(5-6):497504

12. Ye GL, Guo JW, Sun ZZ, Li C, Zhong SY (2018) Weld bead recognition using laser vision with model-based classification. Robot Cim-int Manuf 52:916

13. OConnor S, Clapham L, Wild P (2002) Magnetic flux leakage inspection of tailorwelded blanks. Meas Sci Technol 13(2): 157162

14. Chu HH, Wang ZY (2016) A vision-based system for post-welding quality measurement and defect detection. Int J Adv Manuf Technol 86(9-12):30073014

15. He YS, Chen YX, Xu YL, Huang YM, Chen SB (2016) Autonomous detection of weld seam profiles via a model of saliency-based visual attention for robotic arc welding. J Intell Robot Syst 81(3-4):395406

16. Moon HS, Kim YB, Beattie RJ (2006) Multi sensor data fusion for improving performance and reliability of fully automatic welding system. Int J Adv Manuf Technol 28(3-4):286293

17. Zhang JG, Tan TN (2002) Brief review of invariant texture analysis methods. Pattern Recogn 35(3):735747

18. Bharati MH, Liu JJ, MacGregor JF (2004) Image texture analysis: methods and comparisons. Chemometr Intell Lab Syst 72(1): 5771 
19. Materka A, Strzelecki M (1998) Texture Analysis Methods: A Review. Technical University of Lodz COST B11 Report

20. Mohanaiah P, Sathyanarayana P, GuruKumar L (2013) Image texture feature extraction using glem approach. Int J Sci Res Publ 3(5):15

21. Usamentiaga R, Molleda J, Garca DF (2012) Fast and robust laser stripe extraction for 3D reconstruction in industrial environments. Mach Vision Appl 23(1):179196

22. Sun QC, Chen J, Li CJ (2015) A robust method to extract a laser stripe centre based on grayscale level moment. Opt Laser Eng 67:122127 